“ (C) 2016 IEEE. Personal use of this material is permitted. Permission from IEEE must be obtained for all other uses, in any current or future media, including

reprinting/republishing this material for advertising or promotional purposes, creating new collective works, for resale or redistribution to servers or lists, or reuse of any copyrighted component of this work in other works." 


\title{
ON DIRECTIVITY OF A CIRCULAR ARRAY WITH DIRECTIONAL MICROPHONES
}

\author{
Hongsen He ${ }^{1,2}$, Xiaojun Qiu $^{2}$, and Tao Yang ${ }^{1}$ \\ 1: School of Information Engineering, \\ Robot Technology Used for Special Environment \\ Key Laboratory of Sichuan Province, \\ Southwest University of Science and Technology, \\ Mianyang 621010, China \\ hongsenhe@gmail.com, yangtao98@tsinghua.org.cn \\ 2: Key Laboratory of \\ Modern Acoustics of MOE, \\ Institute of Acoustics, \\ Nanjing University, \\ Nanjing 210093, China \\ xjqiu@nju.edu.cn
}

\begin{abstract}
Circular microphone arrays have a broad range of applications in teleconferencing and hands-free telecommunication systems. Directional microphones are extensively used to construct the circular arrays to obtain superior sound quality. However, the 360-degree coverage ability of this type of circular arrays is seldom investigated. In this paper, we develop a circular array with the use of four firstorder supercardioid microphones to obtain 360-degree coverage of sound recording in teleconferencing environments. Through analyzing the directional response of this array, we derive an optimal range of the array radius for uniform 360-degree coverage of sound capturing. Experiments are carried out in an anechoic chamber to test the performance of the developed microphone array.
\end{abstract}

Index Terms - Circular microphone arrays, supercardioid microphones, directivity.

\section{INTRODUCTION}

Microphone arrays, which are used to capture sound signals, are extensively employed in voice communication systems, noise control systems, and machine learning systems [1]-[3]. Commonly used arrays include linear, circular, planar, spherical, and distributed configurations. Among these configurations, circular arrays can work in full circle range to capture sound signals from 360-degree azimuth, and so they are widely employed in teleconferencing systems. This paper is concerned with this type of arrays.

In teleconferencing systems, some microphone arrays are made up of omnidirectional microphones [1]-[6], which have the same gain at different directions. Another type of arrays uses directional microphones which have large gain at some directions but small gain at other directions [7]. Directional microphones can be viewed as differential array with different orders [8], and widely used such microphones include the types of cardioid, hypercardioid, and supercardioid [9]-[11]. Due to the directivity of directional microphones and significantly superior sound quality provided by arrays using directional microphones [12], this class of microphones can be used by distributed ways to capture and enhance sound signals from different directions. For instance, a popular product in teleconferencing system market is a triangular array with cardioid microphones [13],

This work was supported in part by the National Science Foundation of China (NSFC) (Grant No. 61571376), the Incubation Program for the Distinguished Youth Foundation of Sichuan Province of China (Grant No. 2014JQ0042), the Open Foundation of the Key Laboratory of Modern Acoustics of Nanjing University (Grant No. 1302), the Doctoral Foundation of Southwest University of Science and Technology (Grant No. 13zx7149), and the Open Foundation of Robot Technology Used for Special Environment Key Laboratory of Sichuan Province (Grant No. 13zxtk06). which situates at the center of a conference table to capture the sound signals of voiced participants. However, fewer efforts have been reported to show their performance behavior.

A circular array with hypercardioid microphones was proposed to capture and reproduce interaural time delay and interaural level difference cues that a listener would experience in an actual sound field [14]. This work was extended by De Sena et al. to design a multichannel system for perceptual sound field reconstruction [15]. To obtain a preferable array diameter, the authors analyzed the influence of the diameter of the circular microphone array on the width and naturalness of the reproduced phantom images [16]. However, the 360-degree coverage ability of a circular array with unidirectional microphones in teleconferencing systems was not examined.

In this paper, the 360-degree coverage ability of a circular array with unidirectional microphones is investigated. First, the directivity of the unidirectional microphone is discussed. Then, an equispaced circular array with four supercardioid microphones is constructed. The directional response of this array and the effect of the array radius on its directivity are analyzed. Finally, experiments are carried out in an anechoic chamber to measure the 360-degree coverage ability of the constructed array.

\section{DIRECTIVITY OF A CIRCULAR ARRAY WITH SUPERCARDIOID MICROPHONES}

\subsection{Unidirectional Microphone}

The physical structure of a typical unidirectional microphone is illustrated in Fig. 1 [17]-[19]. This type of microphones is a combination of both the pressure and pressure difference microphones (so, the unidirectional microphones can be viewed as the first-order differential microphones [9], [10]). The diaphragm with equivalent acoustic resistance $Z_{\mathrm{AD}}$ is fixed in front of a cavity $V$ with acoustic capacitance $C_{\mathrm{a}}$. At the back of this cavity, there is a hole as the second acoustic entrance, in which the acoustic material with resistance $R_{\mathrm{a}}$ is filled. The sound pressure of the incidence wave in front of the diaphragm is $p_{1}$, and the angle between the direction of the incidence wave and the vertical line of the diaphragm is $\phi$. The sound pressure of the second acoustic entrance is $p_{2}$. The acoustic path difference between the front of the diaphragm and the acoustic hole at the back of the cavity is denoted as $\Delta$.

Now, if we assume that the sound source is a point source located in the far field, we have

$$
p_{1}=\frac{A}{r} e^{j(\omega t-k r)},
$$




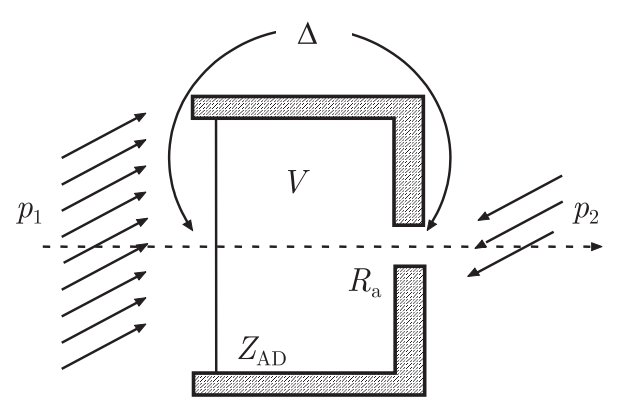

Fig. 1. Physical structure of a unidirectional microphone.

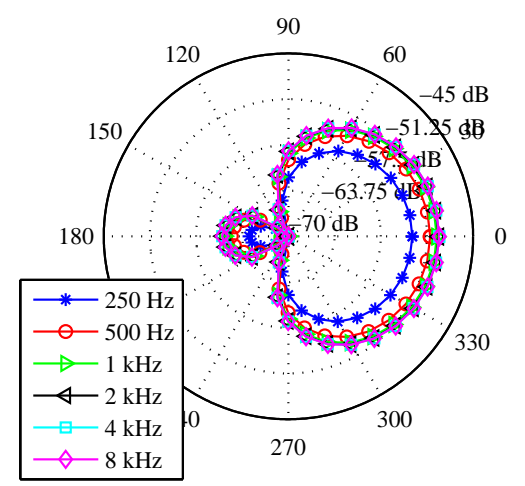

Fig. 2. Directivity pattern of a supercardioid microphone.

where $A$ is some constant, $r$ is the distance from the sound source to the microphone, $j$ is the imaginary unit with $j^{2}=-1, k=\omega / c_{0}$, $c_{0}$ is the sound velocity in air, and

$$
p_{2}=p_{1}+\frac{\partial p_{1}}{\partial r} \Delta \cos \phi=p_{1}(1-j k \Delta \cos \phi) .
$$

The sound pressure difference on both sides of the diaphragm can be derived as

$$
p_{\mathrm{D}_{\mathrm{Mic}}}=\frac{A+B \cos \phi}{1-j C / \omega} \cdot \frac{1}{r} e^{j(\omega t-k r)},
$$

where $B=\frac{A \Delta}{c_{0} C_{\mathrm{a}} R_{\mathrm{a}}}, C=\frac{\beta+1}{C_{\mathrm{a}} R_{\mathrm{a}}}, \beta=\frac{R_{\mathrm{a}}}{Z_{\mathrm{AD}}}$. Then, the amplitude of the sound pressure difference is

$$
\left|p_{\mathrm{D}_{\mathrm{Mic}}}\right|=\frac{\omega}{r \sqrt{\omega^{2}+C^{2}}}(A+B \cos \phi) .
$$

Therefore, the directional response of the unidirectional microphone is

$$
D_{\mathrm{Mic}}(f, \phi)=\frac{2 \pi f}{r \sqrt{4 \pi^{2} f^{2}+C^{2}}}(A+B \cos \phi) .
$$

When the parameters $A$ and $B$ take different values, the directivity pattern of the unidirectional microphones has different shapes, such as cardioid, hypercardioid, supercardioid [9], [10]. For a typical unidirectional microphone such as the supercardioid microphone 9750L [20], the parameters in (5) are chosen as $A=0.0033(\sqrt{3}-$ $1) / 2, B=0.0033(3-\sqrt{3}) / 2$, and $C=1802.4$. The directional response of this type of supercardioid microphones is shown in Fig. 2,

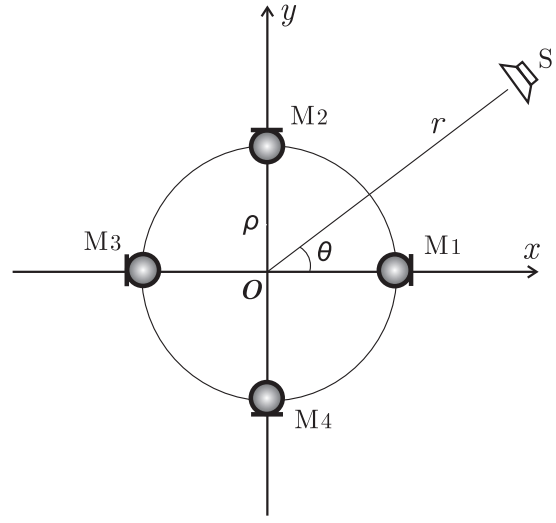

Fig. 3. Configuration of an equispaced circular array with four supercardioid microphones.

from which one can see that this type of microphones has a large gain in front of the diaphragm. A fundamental characteristic of the supercardioid microphones is that its directivity pattern has the maximum front-to-back ratio [9].

\subsection{A Circular Array With Supercardioid Microphones}

Circular microphone arrays, which can capture sounds from 360degree azimuth, are widely applied to hands-free voice communication systems. We use unidirectional microphones to construct a circular array due to superior sound quality provided by the array with the unidirectional microphones [12]. In such an array, each microphone points outward along the radial direction. If the main lobe of the directivity pattern of a unidirectional microphone is sharp enough, multiple such microphones can be used to construct an equispaced circular array to capture sounds from all directions in the form of switching. Consequently, this array has good ability to reduction of spatial noises and reverberation. However, most commercial unidirectional microphones have wide main lobe, such as the first-order cardioid, hypercardioid, and supercardioid microphones [10], [11]. Among the three types of unidirectional microphones, the first-order supercardioid microphones obtain a good compromise between the main lobe width and the front-to-back ratio. So, we choose the first-order supercardioid microphones to construct the circular array.

If the distance from the sound source to the array center is fixed, both the array radius and the number of microphones can affect the array performance. It is, therefore, important to determine an appropriate array radius and number of microphones when one designs the circular array for a teleconferencing system based on the use of unidirectional microphones. For the array radius, we will discuss later on. As to the second concern, since the gain of the first-order supercardioid microphones largely diminishes beyond the main lobe of 120 degrees (which can be seen from Fig. 2) [8], [9], four supercardioid microphones are sufficient to capture sounds from the whole 360-degree azimuth in consideration of some margins to circumvent the sound leakage at the gap between two adjacent main lobes. Our array configuration is shown in Fig. 3. The diaphragms of the microphones $\mathrm{M}_{1}, \mathrm{M}_{2}, \mathrm{M}_{3}$, and $\mathrm{M}_{4}$ point outward along the radial direction, respectively. The array radius is $\rho$. The azimuth angle of the sound source $\mathrm{S}$ is $\theta$. The distance between the sound source and the array center is $r$. 


\subsection{Directional Response of the Circular Array}

In light of the physical structure of unidirectional microphones shown in Fig. 1 and the sound pressure difference in (3), it can be derived from Fig. 3 that the sound pressure difference on both sides of each microphone diaphragm is

$$
p_{\mathrm{D}_{\mathrm{Mi} i}}=\frac{A+B \cos \phi_{i}}{1-j C / \omega} \cdot \frac{1}{r_{i}} e^{j\left(\omega t-k r_{i}\right)}, i=1,2,3,4,
$$

where

$$
\begin{aligned}
& r_{1}=\sqrt{r^{2}+\rho^{2}-2 r \rho \cos \theta}, \\
& r_{2}=\sqrt{r^{2}+\rho^{2}-2 r \rho \cos \left(\frac{\pi}{2}-\theta\right)}, \\
& r_{3}=\sqrt{r^{2}+\rho^{2}-2 r \rho \cos (\pi-\theta)}, \\
& r_{4}=\sqrt{r^{2}+\rho^{2}-2 r \rho \cos \left(\frac{\pi}{2}+\theta\right)},
\end{aligned}
$$

and

$$
\begin{aligned}
& \phi_{1}=\pi-\cos ^{-1}\left(\frac{r_{1}^{2}+\rho^{2}-r^{2}}{2 r_{1} \rho}\right), \\
& \phi_{2}=\pi-\cos ^{-1}\left(\frac{r_{2}^{2}+\rho^{2}-r^{2}}{2 r_{2} \rho}\right), \\
& \phi_{3}=\pi-\cos ^{-1}\left(\frac{r_{3}^{2}+\rho^{2}-r^{2}}{2 r_{3} \rho}\right), \\
& \phi_{4}=\pi+\cos ^{-1}\left(\frac{r_{4}^{2}+\rho^{2}-r^{2}}{2 r_{4} \rho}\right) .
\end{aligned}
$$

To capture the voiced signals by this circular array, it is advisable to emphasize the signals of some microphones facing to voiced participants while attenuate those of other microphones facing to unvoiced participants. To this end, the sound pressure difference on both sides of each microphone diaphragm is added in some weighting way to obtain a total one, i.e.,

$$
\begin{aligned}
p_{\mathrm{D}_{\mathrm{Array}}} & =\sum_{i=1}^{4} w_{i} p_{\mathrm{D}_{\mathrm{Mic} i}} \\
& =\sum_{i=1}^{4}\left[w_{i} \frac{A+B \cos \phi_{i}}{1-j C / \omega} \cdot \frac{1}{r_{i}} e^{j\left(\omega t-k r_{i}\right)}\right] .
\end{aligned}
$$

where $w_{i}(i=1,2,3,4)$ is the weighting coefficient corresponding to channel $i$, which can be determined by comparison of the shorttime energy between all the channels [21]. In this paper, we focus attention on the investigation into the 360-degree coverage ability of the directional response of the array, and so the coefficient $w_{i}(i=$ $1,2,3,4)$ is set to 1.0. If the time variable $t$ is omitted, we can obtain

$$
p_{\mathrm{D}_{\text {Array }}}=\sum_{i=1}^{4}\left(\frac{A+B \cos \phi_{i}}{1-j C / \omega} \cdot \frac{1}{r_{i}} e^{-j k r_{i}}\right) .
$$

Then, we achieve the directional function of the circular array with four supercardioid microphones as

$$
D_{\text {Array }}(f, \theta)=\left|\sum_{i=1}^{4}\left[\frac{A+B \cos \phi_{i}}{1-j C /(2 \pi f)} \cdot \frac{1}{r_{i}} e^{-j 2 \pi f r_{i} / c_{0}}\right]\right| .
$$

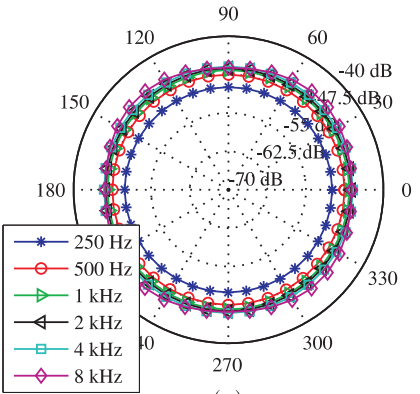

(a)

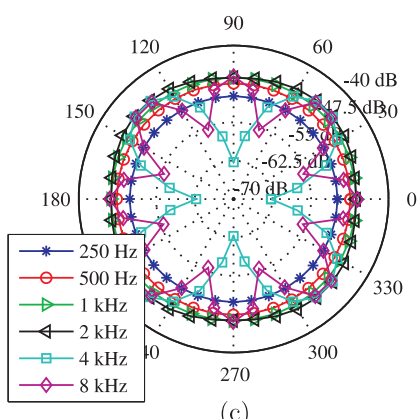

(c)

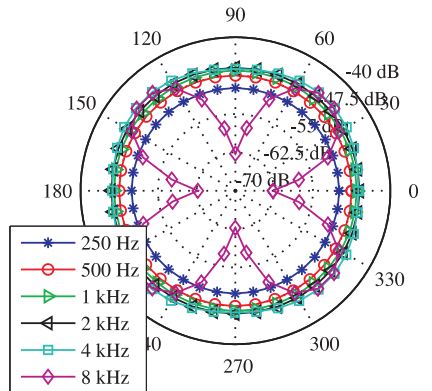

(b)

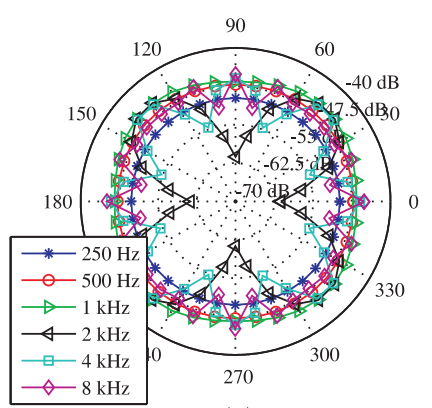

(d)
Fig. 4. Directivity pattern of the circular array with the radiuses of (a) $1 \mathrm{~m}$, (b) $2 \mathrm{~m}$, (c) $4 \mathrm{~m}$, and (d) $8 \mathrm{~m}$.

It can be seen from (11) that the array radius has an effect on the directional response of the array. The directivity pattern of the array with different radiuses is shown in Fig. 4, from which it can be seen that as the array radius is increased, the directivity of the array at the whole 360-degree azimuth is more and more uneven. For low and moderate frequencies, the array gain at the whole 360-degree azimuth is basically equal.

\section{EXPERIMENTS}

\subsection{Experimental Environments}

Experiments are carried out in the anechoic chamber at Nanjing University of China to validate the directional response of the proposed circular array. The temperature and humidity are $10{ }^{\circ} \mathrm{C}, 88 \%$, respectively. Four supercardioid microphones with type $9750 \mathrm{~L}$ are employed to construct the circular array, as shown in Fig. 3. The gain and phase of these microphones are calibrated. The distance from the sound source to the array center is $1 \mathrm{~m}$. The B\&K Pulse 3560 and Turntable 5960 are used to measure the directivity pattern of the microphone and array.

\subsection{Results}

First, the directivity pattern of the supercardioid electret microphone with type $9750 \mathrm{~L}$ is measured. The comparison between the simulation and experimental results is shown in Fig. 5(a), from which it can be seen that the directional response from the simulations coincides with the measurement results for the frequencies under $4 \mathrm{kHz}$.

Figs. 5(b) through (d) make a comparison of the directivity pattern of the circular array between the simulations and experiments when the array radius is $2 \mathrm{~cm}, 4 \mathrm{~cm}$, and $8 \mathrm{~cm}$, respectively. We 


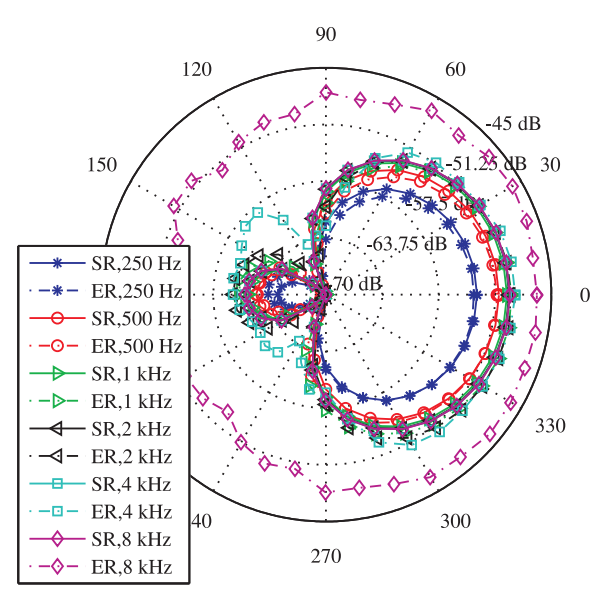

(a)

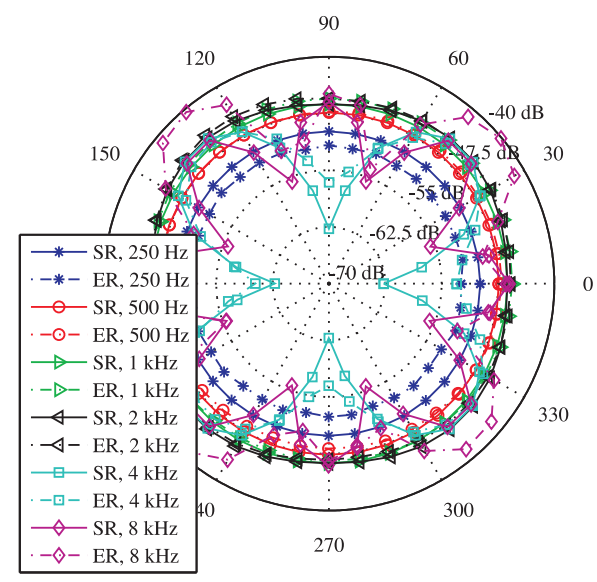

(c)

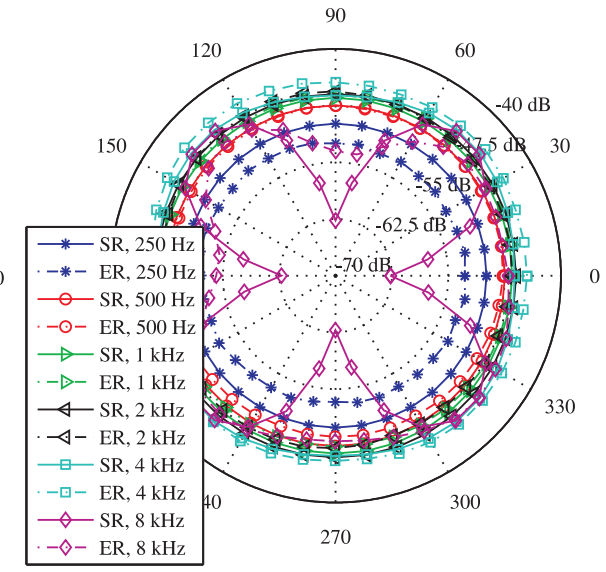

(b)

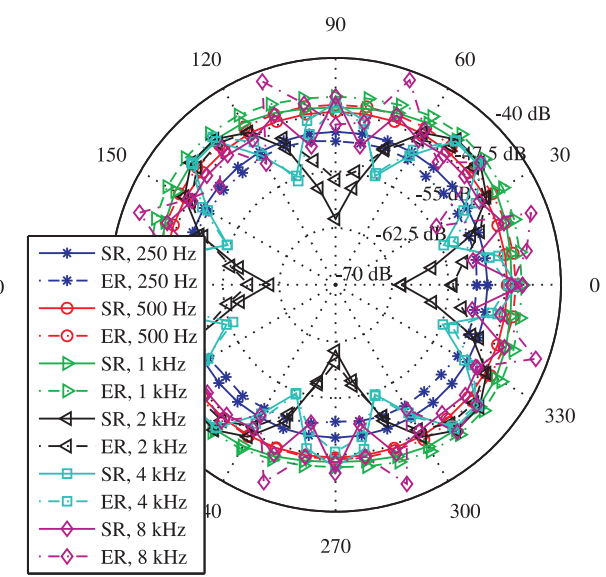

(d)

Fig. 5. Comparison of the directivity pattern of the supercardioid microphone and circular array with different radiuses between simulations and experiments, where SR denotes the simulation results and ER stands for the experimental results. Plot (a) depicts the supercardioid microphone; plots (b), (c), and (d) illustrate the circular array with the radiuses of $2 \mathrm{~m}, 4 \mathrm{~m}$, and $8 \mathrm{~m}$, respectively.

can see that when the array radius is $2 \mathrm{~cm}$ the experimental results are similar to the simulation results for the frequencies from 500 $\mathrm{Hz}$ to $4 \mathrm{kHz}$. When the array radius is increased, the directional responses from the experiments are basically comparable to those from the simulations for low and moderate frequencies. It can also be seen that when the array radius is small, the directional responses for the typical frequencies of speech signals are basically a circle, which indicates that a small array radius is preferable for even 360-degree coverage of sound capturing.

\section{CONCLUSIONS}

Microphone arrays are extensively employed to capture sounds in hands-free communication systems. Unidirectional microphones are commonly used to form microphone arrays for superior sound quality. In this paper, an equispaced circular array with four supercardioid microphones was constructed. The directional response of this array and the effect of the array radius on its directivity were investigated. Experiments were carried out in an anechoic chamber. The results demonstrate that a small array radius is preferable for even 360-degree coverage of sound capturing. When the array radius is less than $4 \mathrm{~cm}$, the array obtains even gain at the whole 360-degree azimuth for the dominant frequencies of speech signals.

\section{ACKNOWLEDGMENTS}

The authors would like to thank Prof. Jingdong Chen of Northwestern Polytechnical University of China for his valuable suggestions and revision on this paper.

\section{REFERENCES}

[1] M. S. Brandstein and D. B. Ward, Microphone Arrays: Signal Processing Techniques and Applications. Berlin, Germany: Springer-Verlag, 2001.

[2] J. Benesty, J. Chen, and Y. Huang, Microphone Array Signal Processing. Berlin, Germany: Springer-Verlag, 2008.

[3] J. Benesty, M. M. Sondhi, and Y. Huang, Springer Hand- 
book of Speech Processing. Berlin, Germany: Springer-Verlag, 2008.

[4] J. Chen, J. Benesty, Y. Huang, and S. Doclo, "New insights into the noise reduction Wiener filter," IEEE Trans. Audio, Speech, Lang. Process., vol. 14, no. 4, pp. 1218-1234, Jul. 2006.

[5] H. He, L. Wu, J. Lu, X. Qiu, and J. Chen, “Time difference of arrival estimation exploiting multichannel spatio-temporal prediction," IEEE Trans. Audio Speech Lang. Process., vol. 21, no. 3, pp. 463-475, Mar. 2013.

[6] H. He, J. Lu, J. Chen, X. Qiu, and J. Benesty, "Robust blind identification of room acoustic channels in symmetric alphastable distributed noise environments," J. Acoust. Soc. Amer., vol. 136, no. 8, pp. 693-704, Aug. 2014.

[7] C. Zhang, D. Florencio, D. Ba, and Z. Zhang, "Maximum likelihood sound source localization and beamforming for directional microphone arrays in distributed meetings," IEEE Trans. Multimedia, vol. 10, no. 3, pp. 538-548, Apr. 2008.

[8] G. W. Elko, "Microphone array systems for hands free telecommunications," Elsevier Speech Commun., vol. 20, pp. 229-240, Sep. 1996.

[9] G. W. Elko, "Superdirectional microphone arrays," in Acoustic Signal Processing for Telecommunication, S. L. Gay and J. Benesty, Eds. Norwell, MA: Kluwer, 2000.

[10] I. J. Tashev, Sound Capture and Processing: Practical Approaches. Chichester, UK: Wiley, 2009.

[11] J. Benesty and J. Chen, Study and Design of Differential Microphone Arrays. Berlin, Germany: Springer-Verlag, 2012.

[12] I. Tashev and H. Malvar, "A new beamformer design algorithm for microphone arryas," in Proc. IEEE Int. Conf. Acoust., Speech, Signal Process.(ICASSP), 2005.

[13] [Online]. The conference phone VoiceStation 300 of the Polycom Inc. Available: http://www.polycom.com/productsservices/voice/confer-encing-solutions/conferencingphones/voicestation-300.html, 2009.

[14] J. D. Johnston and Y. H. Lam, "Perceptual soundfield reconstruction," in Proc. Audio Eng. Soc. (AES) 109th Conv., 2000, Preprint \#2399.

[15] E. De Sena, H. Hacihabiboglu, and Z. Cvetkovic, "Analysis and design of multichannel systems for perceptual sound field reconstruction," IEEE Trans. Audio Speech Lang. Process., vol. 21, no. 8, pp. 1653-1665, Aug. 2013.

[16] E. De Sena, H. Hacihabiboglu, and Z. Cvetkovic, "Design of a circular microphone array for panoramic audio recording and reproduction: Array radius," in Proc. Audio Eng. Soc. (AES) 128th Conv., 2010, Preprint \#8064.

[17] B. Bauer, "A Century of Microphones," Proc. IRE, vol. 50, no. 5, pp. 719-729, May 1962.

[18] G. Du, Z. Zhu, and X. Gong, Fundamentals of Acoustics (in Chinese). Nanjing, China: Nanjing University Press, 2001.

[19] J. Eargle, The Microphone Book, 2nd ed. Oxford: Focal Press, 2005.

[20] [Online]. The typical supercardioid microphone with type $9750 \mathrm{~L}$ of the Gettop Co.. Available: http://www.gettopacoustic.com/en/product151.html, 2009.

[21] H. He and X. Qiu, "Speaker tracking with a circular array of directional microphones," in Proc. IEEE Int. Conf. Comput. Sci. Autom. Eng. (ICCSAE), 2013. 